Strahlenther Onkol 2012.

[Suppl 3] ·188:219-219

DOI 10.1007/s00066-012-0184-2

Online publiziert: 22. August 2012

๑) Springer-Verlag Berlin Heidelberg 2012

J. Dunst
Klinik für Strahlentherapie, Universität zu Lübeck

\title{
Grußwort des Präsidenten der Deutschen Gesellschaft für Radioonkologie
}

Strom (Zytostatika gab es noch nicht). Herr PD Dr. Werner aus Heidelberg schreibt in seiner Arbeit über Behandlung der malignen Tumoren: „Nach den Tierversuchen sind nämlich die Krebszellen gegen bestimmte Hitzegrade, die um $50-55^{\circ} \mathrm{C}$ liegen, empfindlicher als die normalen Körperzellen."

Das Vermeiden von Nebenwirkungen spielt schon in den ersten Arbeiten eine wichtige Rolle. Methoden zum Schutz der nicht bestrahlten Körperregionen werden beschrieben. Die Diskrepanz zwischen akuten und chronischen Strahlenfolgen wird diskutiert. Einerseits war schon bekannt, dass bei Überschreiten der „Erythemdosis“ schwere und bleibende, verbrennungsartige Hautreaktionen resultieren. Andererseits waren Fälle bekannt, in denen Hautareale, die bereits ein Erythem gezeigt hatten, vollständig abheilten und später, nach langem Intervall, nochmals bestrahlt werden konnten. Auch das Problem der Sekundärmalignome war bereits 1912 (16 Jahre nach der ersten Strahlenanwendung!) bekannt. Herr PD Dr. Hans Meyer aus Kiel, der erste habilitierte Strahlentherapeut in Deutschland und Mitbegründer der Zeitschrift, weist in einer Arbeit über die Methodik der Röntgentherapie in der Gynäkologie darauf hin: „Wir wissen heute, dass schon nach einer einzigen schweren Reaktion der Haut, also nach einer heftigen Röntgendermatitis, sich noch nach Jahren der Zustand der Röntgenatrophie der Haut einstellen kann, ein Ereignis, dass nicht nur aus kosmetischen Gründen vermieden werden muss, sondern vor allem auch deswegen unsere volle Beachtung verdient, weil diese röntgenatro- phischen Stellen, wie die Erfahrung lehrt, wenn auch nur in seltenen Ausnahmefällen, zu malignen Neubildungen prädisponieren können."

Die erste Arbeit in der Zeitschrift (also Seite 1, Heft 1, Band 1) stammt von Hofrat Prof. v. Noorden aus Wien und lautet: „Die Bedeutung der Therapie mit radioaktiven Substanzen für die innere Medizin“. Am Ende der Arbeit kommt er zu folgenden Schlussfolgerungen: „Wir stehen erst am Anfang der radioaktiven Therapie. Wir werden sicher noch manche Enttäuschungen, aber sicher auch manche freudige Überraschung erleben. Die radioaktive Therapie soll und wird andere bewährte Heilmethoden nicht verdrängen, aber sie wird sicher neben ihnen einen angesehenen und allgemein anerkannten Platz erobern. Ehe dieses Ziel erreicht ist, steht noch viel Arbeit vor uns. Wir werden umso schneller und umso sicherer zu ihm gelangen, wenn wir einstweilen mehr die biologischen und pharmakologischen Voraussetzungen derselben als die unsicheren, dem subjektiven Urteil nicht entzogenen und nur durch breiteste und lange Erfahrung sicher zu stellenden unmittelbaren therapeutischen Wirkung studieren." Er hatte damals und hat auch noch heute Recht!

Prof. Dr. Jürgen Dunst

\section{Korrespondenzadresse}

Prof. Dr. J. Dunst

Universität zu Lübeck

Lübeck

Deutschland

juergen.dunst@uksh.de rere Arbeiten mit der Modifizierung der Strahlenwirkung durch Hitze, Kälte oder 